\title{
Identification and Evolution of the WUSCHEL- Related Homeobox Protein Family in Bambusoideae
}

\author{
Xiangyu Li $®$, Juan Li, Miaomiao Cai, Huifang Zheng, Zhanchao Cheng and Jian Gao * \\ International Center for Bamboo and Rattan, Key Laboratory of Bamboo and Rattan Science and Technology, \\ State Forestry and Grassland Administration, Beijing 100102, China; lxy@icbr.ac.cn (X.L.); lijuan@icbr.ac.cn (J.L.); \\ cmm@icbr.ac.cn (M.C.); zhenghuifang@icbr.ac.cn (H.Z.); czc@icbr.ac.cn (Z.C.) \\ * Correspondence: gaojian@icbr.ac.cn or gaojianicbr@163.com; Tel.: +86-010-8478-9801
}

Received: 25 February 2020; Accepted: 29 April 2020; Published: 9 May 2020

\begin{abstract}
Bamboos (Bambusoideae) are fast-growing species due to their rapid growth rate and ability to reproduce annually via cloned buds produced on the rhizome. WUSCHEL-related homeobox (WOX) genes have been reported to regulate shoot apical meristem organization, lateral organ formation, cambium and vascular proliferation, and so on, but have rarely been studied in bamboos. In this study, the WOXs of both herbaceous bamboo species (12 OlaWOXs and nine RguWOXs) and woody bamboo species (18 GanWOXs, 27 PheWOXs, and 26 BamWOXs) were identified and categorized into three clades based on their phylogenetic relationship-ancient, intermediate, or WUS clade. Polyploidy is the major driver of the expansion of the bamboo WOX family. Eight conserved domains, besides the homeodomain, were identified by comparatively analyzing the WOXs of dicot and monocot species. Intensive purifying selection pressure in the coding region of specific domains explained the functional similarity of WOXs between different species. For Bambusoideae WOXs, polyploidy is the major driver of the expansion of the WOX family. Stronger purifying selection was found in orthologous WOXs of Bambusoideae, especially for WOX4s and WOX5s, which are conserved not only at the translational levels, but also at the genome level. Several conserved cis-acting elements were discovered at similar position in the promoters of the orthologous WOXs. For example, AP2/ERF protein-binding elements and B3 protein-binding elements were found in the promoters of the bamboo WOX4, while MYB protein-binding elements and Dof protein-binding elements were found in the promoters of bamboo WOX5, and MADS protein-binding sites was found in the promoters of bamboo WUS, WOX3, and WOX9. These conserved positions may play an important role in regulating the expression of bamboo WOXs. Our work provides insight into the origin and evolution of bamboo WOXs, and will facilitate functional investigations of the clonal propagation of bamboos.
\end{abstract}

Keywords: bambusoideae; WOXs; polyploidization; selection pressure

\section{Introduction}

Bamboos are among the fastest-growing plants on Earth, and the nearly 1500 described bamboo species are native to all continents except Antarctica and Europe [1,2]. As a potential lignocellulosic material, due to its availability, good material properties, and high yield resources, bamboo is as an important pillar of the forest industry for many Asian countries, as well as some in Africa and Latin America. Bamboo contributes great economic and ecological benefits to the world as a fast-growing wood, as well as a source of food and an industrial material. Bamboo rarely flowers, with flowering cycles of up to 120 years [3]. Bamboo forests mainly spread by clonal reproduction, relying on the meristem of the nutritive organ to create shoots of new individuals. Taking the study of the most economically and ecologically rewarding bamboo, moso bamboo (Phyllostachys edulis), as an example, 
underground growth includes the extension of the rhizome, the development of the rhizome root, and the development of lateral buds located in the internode of the rhizome. The development of lateral buds is extraordinarily important for bamboo development, which proceeds from the dormant buds to the active buds, and then to new shoot development [4]. It takes a long time, even years, for dormant bud development to provide active shoots underground. However, the aboveground growth, from shoots breaking through the soil to young bamboo, finishes in two months, with a high growth rate that is as fast as $1 \mathrm{~m}$ per day [5], which illustrates how rapidly bamboo forests can spread.

Bamboos fall into four monophyletic lineages with different ploidy levels according to the number of basic sets of bamboo chromosomes, diploid herbaceous bamboos $(2 n=20-24)$, temperate tetraploid woody bamboos $(2 n=46-48)$, neotropical tetraploid woody bamboos $(2 n=40-48)$, and palaeotropical hexaploid woody bamboos $(2 n=70-72)$ (reviewed in [1]). Recently, Guo et al. (2019) reported the draft genomes of two diploid herbaceous bamboo species (Olyra latifolia, $2 \mathrm{n}=2 \mathrm{x}=22$; Raddia guianensis, $2 \mathrm{n}=2 \mathrm{x}=22$ ), one neotropical tetraploid woody bamboo (Guadua angustifolia, $2 \mathrm{n}=4 \mathrm{x}=46$ ), and one palaeotropical hexaploid woody bamboo (Bonia amplexicaulis, $2 \mathrm{n}=6 \mathrm{x}=72$ ). A distinct subgenome was identified in these polyploid and Phyllostachys edulis (temperate tetraploid woody bamboo, $2 \mathrm{n}=4 \mathrm{x}=48$ ). The polyploidizations of woody bamboos gave rise to four distinctly ancestral subgenomes, namely, A, B, C, and D, resulting in the allohexaploid palaeotropical Bonia amplexicaulis (AABBCC), the allotetraploid neotropical Guadua angustifolia (BBCC), and the allotetraploid temperate Phyllostachys edulis (CCDD) [1]. This significant research provides the molecular evidence for the insights into the origin of woody bamboos at the subgenome level, and the basis to study gene family evolution during allopolyploidization.

The WUSCHEL-related homeobox (WOX) genes belong to the homeobox transcription factor (HB TF) family, which encode typical plant transcription factors sharing a DNA-binding domain with a conserved stretch of 60-66 residues, the Helix-loop-helix-turn-helix structure [6-8]. The other regions of the WOX proteins are highly divergent [9], on account of which WOX proteins can be divided into three separate clades, namely the ancient clade, the intermediate clade, and the WUS clade [8]. In Arabidopsis, the WOX family consists of 15 members, namely, WUSCHEL (WUS) and WOX1-WOX14 [10]. In Arabidopsis, the ancient clade harbors WOX10, 13, and 14 proteins, the WUS clade harbors WUS and WOX 1-7 proteins, and the intermediate clade harbors WOX8, 9, 11, and 12 proteins.

In vascular plants, WOXs play important roles in various processes, including maintenance of the root and shoot apical meristems, vascular development, embryogenesis development, and adventive organ development [10-13]. WUS, WOX4, and WOX5 regulate stem cell maintenance in the shoot and floral meristems, cambium, and root meristem [11,14-18], and WOX3 contributes to marginal and plate meristem activity of leaves [19,20]. WOX8 (STIMPY-LIKE, STPL) and WOX9 (STIMPY, STIP) of Arabidopsis thaliana are essential for embryo patterning and vegetative SAM maintenance $[10,21,22]$. WOX2 in Arabidopsis thaliana promotes cotyledon boundary formation during early embryogenesis, redundantly with WOX8 [22]. WOX11/12 activate the expression of WOX5 for root primordia initiation during de novo organogenesis $[23,24]$. In Arabidopsis, WUSCHEL (WUS) maintains the stem cell niche in the shoot apical meristem (SAM) by partnering with the WUS-CLAVATA3 (WUS-CLV3) signaling pathway $[12,14,25,26]$. However, the rice ortholog of WUS, TILLERS ABSENT1 (TAB1), is not expressed in the SAM and does not participate in stem cell proliferation, but is transiently expressed in the early stages of axillary meristem development and plays a crucial role by interacting with the CLAVATA3 orthologous gene, FLORAL ORGAN NUMBER2 (FON2), in rice axillary meristem development $[13,17]$. WOX4 of rice acts as a positive regulator of stem cell maintenance in the SAM and axillary meristem [18]. Taken together, the WOX family is essential for the establishment of plant architecture in various fundamental aspects, including the formation and maintenance of shoot and root apical meristems, the development of lateral organs, the maintenance and differentiation of cambium, floral formation, and the formation of polarity in the embryo.

WOX genes may play key roles during bamboo rhizome bud formation, as well as in the activation of dormant buds, the maintenance of shoot apical meristems, and the formation of adventitious roots 
during bamboo clonal reproduction, while the regulatory mechanisms underlying when and how meristems are established, maintained, and lost during bamboo regeneration are poorly understood. In the present study, we identified 91 putative bamboo WOXs of herbaceous and woody bamboos. Phylogenetic analysis classified these WOXs into three clades. Specific motifs were identified by a comparative analysis of the proteins of Phyllostachys edulis (P. edulis) with Arabidopsis thaliana, Populus trichocarpa, Oryza sativa, Zea mays, and Brachypodium distachyon. The conserved specific domain of each clade was characterized and analyzed conjointly with phylogenetic analysis. Comparative analysis of PheWOXs with WOXs of two herbaceous bamboo species and three woody bamboo species was performed. Our study will serve as a foundation for future research on the functional roles of WOXs in Bambusoideae, as well as provide insight into the essential roles of WOXs in P. edulis development.

\section{Methods}

\subsection{Identification of the WOX Gene Family in Bambusoideae Genomes}

WOXs of Arabidopsis thaliana, Populus trichocarpa, Oryza sativa, Zea mays, Brachypodium distachyon, Micromonas pusilla, Micromonas sp., Ostreococcus tauri, and Bathycoccus prasinos were collected from PlantTFDB v4.0 (http://planttfdb.cbi.pku.edu.cn/family.php?fam=WOX) [27] and rechecked by NR (non-redundant protein sequence database) annotation and conserved homeodomain (HD) identification. WOXs from these species were used as queries to blast the $P$. edulis polypeptide dataset (ftp://parrot.genomics.cn/gigadb/pub/10.5524/100001_101000/100498/), the Olyra latifolia (O. latifolia) polypeptide dataset, the Raddia guianensis (R. guianensis) polypeptide dataset, the Guadua angustifolia (G. angustifolia) polypeptide dataset, and the Bonia amplexicaulis (B. amplexicaulis) polypeptide dataset (http://www.genobank.org/bamboo) with the threshold e-value of $10^{-5}[1,28]$. The conserved domain database of NCBI was used to confirm the HD of putative WOX proteins [29]. Furthermore, the genome sequence of putative WOXs was obtained according to Gene ID of the genome information by TBtools [30].

\subsection{Phylogenetic Tree Construction and Conserved Motif Analysis of WOXs}

The multiple alignment of WOX proteins was performed using CLUSTAL_X software [31]. After manually removing the poorly aligned sequences and divergent regions of the WOX protein alignment, MEGA5 was used to construct a neighbor-joining tree of WOXs with a bootstrap assessment of 1000 replicates [32]. A maximum-likelihood tree was also constructed to validate the topologies. Multiple Em for Motif Elicitation (MEME Version 5.0.4, http://meme-suite.org/tools/meme) was used to identify conserved motifs among WOXs using the following parameters: the site distribution of zero or one site per sequence; motifs with a maximum number of 11; and motifs with a width of around 10-70 characters. The visualization of the tree and motif distribution was implemented by Evolview v2.0 [33].

\subsection{Selective Pressure Analysis of Orthologous and Paralogous Genes}

The non-synonymous substitution rate $(\mathrm{Ka})$, the synonymous substitution rate (Ks), and the $\mathrm{Ka} / \mathrm{Ks}$ of gene pairs were calculated using PAML [34]. To identify signals of positive selection of certain regions, a sliding window analysis of the $\mathrm{Ka} / \mathrm{Ks}$ ratios was carried out with a window size of 60 Angstrom using SWAKK [35]. The divergence time of the homologous WOXs was calculated using the formula: $\mathrm{T}=\mathrm{Ks} / 2 \lambda$ [36], with the divergence rate $\lambda=6.5 \times 10^{-9}$ [2].

\section{4. cis-Acting Element Analysis of the P. edulis WOX Promoter}

The WOX promoter sequence $1500 \mathrm{bp}$ upstream from the start codon (ATG) was extracted using TBtools [30]. The cis-acting element analysis was conducted based on the cis-acting element dataset of Plant Transcriptional Regulatory Map (PlantRegMap), which was derived from high-throughput 
assays of ChIP-seq and genome-wide TF footprinting [27,37]. The regulatory element distribution of the WOX promoter was analyzed with the threshold $p$-value of $10^{-5}$.

\section{Results}

\subsection{Identification of the WOXs of Bambusoideae}

Twenty-seven putative WOX genes of Phyllostachys edulis (PheWOXs) were identified in the P. edulis genome (Version 2) (Supplementary Table S1) [28]. Of these, 21 contain complete HDs with around 60 amino acid residues, and the other six code proteins with incomplete HDs at the N-terminal region which may impede DNA-binding, but show high similarity with WOX [6,8]. In addition, the WOX genes of four other bamboos (O. latifolia, R. guianensis, G. angustifolia, and B. amplexicaulis) were also identified from draft genomes [1], namely 12 WOXs of O. latifolia (OlaWOXs), nine WOXs of R. guianensis (RguWOXs), 18 WOXs of G. angustifolia (GanWOXs), and 26 WOXs of B. amplexicaulis (BamWOXs) (Supplementary Table S1). The number of ortholog of WOX is weakly correlated with Bambusoideae ploidy (Table 1). Polyploidy is a major driver of plant evolution [38], and drives family expansion and functional divergence of WOXs. The number of WOX orthologs generally increases with ploidy level as would be expected with increase genome copies. The single exception is hexaploid $B$ amplexiaulis with 26 WOX orthologs compared to 27 orthologs in the tetraploid genome of $P$. edulis. This lower than expected number is likely due to the lower sequence quality and assembly of B. amplexicaulis (848 $\mathrm{Mb}$ of scaffold with an $\mathrm{N} 50$ length of $1.76 \mathrm{Mb}$ ) compared to P. edulis (1886 Mb of scaffold with an N50 length of $79.90 \mathrm{Mb})[1,28]$.

Table 1. Comparison of the WOXs of Bambusoideae with other species.

\begin{tabular}{|c|c|c|c|c|c|c|}
\hline $\begin{array}{l}\text { Taxonomic } \\
\text { Group }\end{array}$ & Species & $\begin{array}{l}\text { Ancient } \\
\text { Clade }\end{array}$ & $\begin{array}{c}\text { Intermediate } \\
\text { Clade }\end{array}$ & $\begin{array}{l}\text { WUSCHEL } \\
\text { (WUS) Clade }\end{array}$ & Other & Total \\
\hline \multirow{2}{*}{ Dicots } & Arabidopsis thaliana & 3 & 4 & 8 & - & 15 \\
\hline & Populus trichocarpa & 3 & 4 & 11 & - & 18 \\
\hline \multirow{8}{*}{ Monocots } & Oryza sativa & 1 & 6 & 6 & - & 13 \\
\hline & $\begin{array}{l}\text { Brachypodium } \\
\text { distachyon }\end{array}$ & 2 & 5 & 6 & - & 13 \\
\hline & Zea mays & 3 & 7 & 10 & - & 20 \\
\hline & Olyra latifolia & 1 & 5 & 6 & - & 12 \\
\hline & Raddia guianensis & 1 & 3 & 5 & - & 9 \\
\hline & Guadua angustifolia & 2 & 9 & 7 & - & 18 \\
\hline & Phyllostachys edulis & 2 & 11 & 14 & - & 27 \\
\hline & Bonia amplexicaulis & 2 & 6 & 18 & - & 26 \\
\hline \multirow{4}{*}{ Chlorophyta } & Micromonas pusilla & - & - & - & 4 & 4 \\
\hline & Micromonas sp. & - & - & - & 1 & 1 \\
\hline & Ostreococcus tauri, & - & - & - & 1 & 1 \\
\hline & Bathycoccus prasinos & - & - & - & 1 & 1 \\
\hline
\end{tabular}

The WOX proteins of Arabidopsis thaliana, Populus trichocarpa, Oryza sativa, Zea mays, and Brachypodium distachyon were also collected from PlantTFDB v4.0 and manually examined (Supplementary Table S2). After removing the redundant sequences with the same chromosome locations, 15 WOXs of Arabidopsis thaliana (AtWOXs), 18 WOXs of Populus trichocarpa (PotriWOXs), 13 WOXs of Oryza sativa (OsWOXs), 20 WOXs of Zea mays (ZmWOXs), and 13 WOXs of Brachypodium distachyon (BradiWOXs) were used for later analysis. All of the candidate WOX proteins contained a 
domain matching IPR001356 (homeobox domain, HD) of the Interpro database. The number of WOXs varied in different species (Table 1).

The nomenclature of the WOXs genes varies among different species, resulting in confusion regarding the understanding of gene function. For example, Os03g0325600 of rice was named both WOX6 and WOX12B (https://rapdb.dna.affrc.go.jp/viewer/gbrowse_details/irgsp1?name=Os03g0325600). In this study, the WOXs were named after their orthologs of AtWOXs based on the conserved domain of the different clades and their evolutionary relationships. The notations $\mathrm{a}, \mathrm{b}$, and $\mathrm{c}$ were appended to differentiate highly similar paralogs in the same species (Supplementary Table S1).

\subsection{Identification of Conserved Domains of the WOX Family}

To better understand the protein features of WOXs, the conserved motifs of these WOXs were searched by MEME. Eleven conserved domains were found among the 171 WOX proteins (Figure 1). Motifs 1-3 are residues of HDs that contain three helices spaced by one loop and one turn [6]. Conserved amino acids of HDs [6,9,39], Q, L, and E of helix 1, P, I, and L of helix 2, N, V, W, F, Q, N, and R of helix 3 , and $\mathrm{G}$ of the turn were identified (Figure 1). In addition to HDs, eight specific domains were found across the WOXs of dicots and monocots, including Motifs 4-6 found in the ancient clade, Motifs 7-10 found in the intermediate clade, and Motif 11 found in the WUS clade (Figure 1). Most of the members of each clade share one or more conserved domains, in addition to HDs (Figure 2). These domains were conserved during evolution of the family and may play a particular role in protein function.

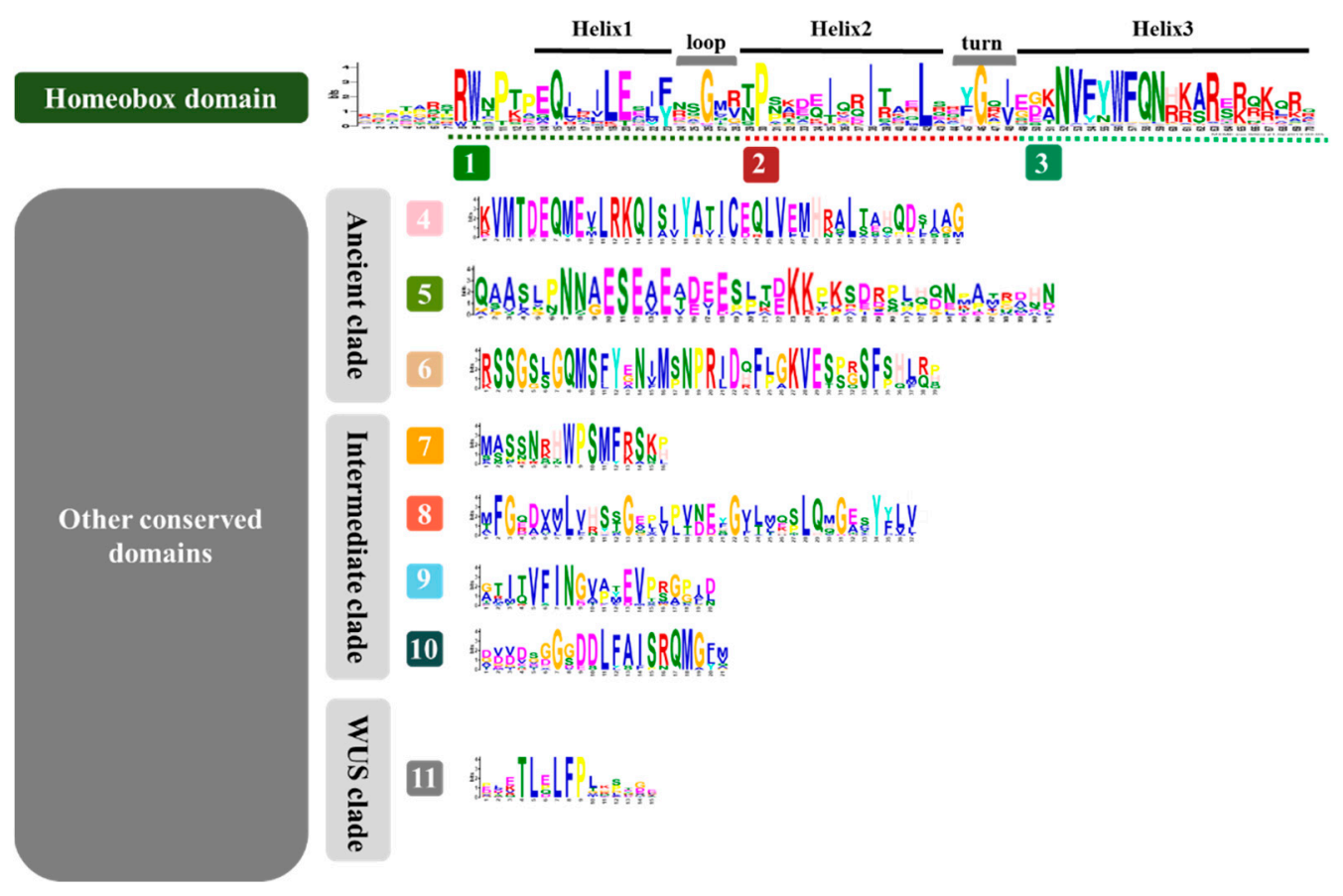

Figure 1. The conserved domains identified in the WUSCHEL-related homeobox (WOX) family. Eleven conserved domains were identified among 15 AtWOXs, 18 PotriWOXs, 13 OsWOXs, 20 ZmWOXs, 27 PheWOXs, 13 BradiWOXs, 12 OlaWOXs, 9 RguWOXs, 18 GanWOXs, and 26 BamWOXs by Multiple Em for Motif Elicitation (MEME). Motifs 4-6 were identified in the WOXs belonging to the ancient clade. Motifs 7-10 were identified in the WOXs belonging to the intermediate clade. Motif 11 was found in the WOX belonging to the WUS clade. 


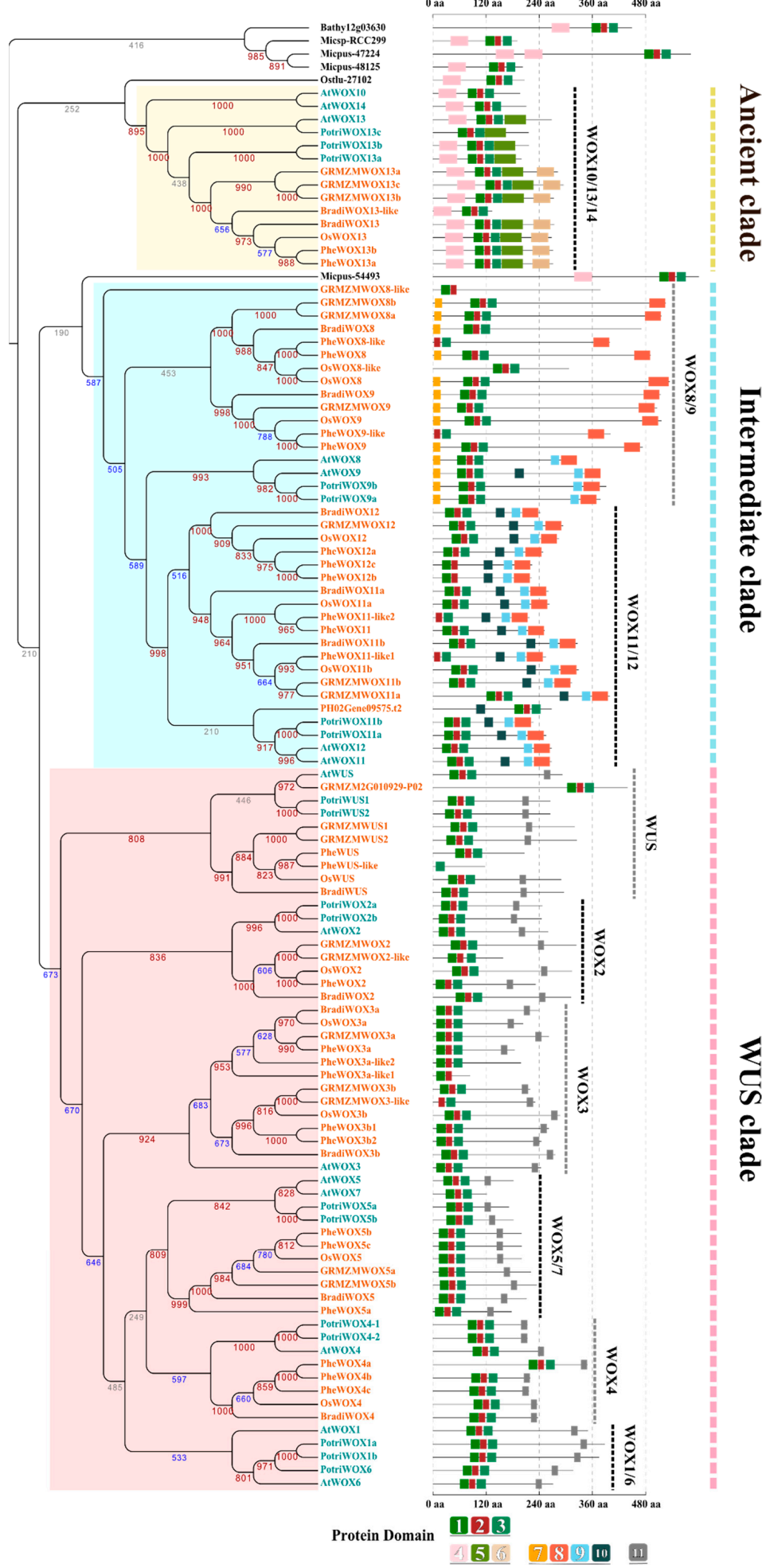

Figure 2. The phylogenetic tree of WOXs from dicots and monocots. The neighbor-joining tree was constructed using amino acid sequences of WOXs from Arabidopsis thaliana (At), Populus trichocarpa (Potri), Oryza sativa (Os), Zea mays (Zm), Brachypodium distachyon (Bradi), Micromonas pusilla (Micpu), Micromonas sp. (Micsp), Ostreococcus tauri, (Osta), and Bathycoccus prasinos (Bathy), with bootstrap assessment of 1000 replicates. Each clade was assigned a different colored background. Leaf colors of black, orange, and cyan stand for WOXs of chlorophytes, monocots, and dicots, respectively. The graphic symbols of the conserved motifs are colored and termed the same as in Figure 1. 


\subsection{The Evolution of the WOX Family}

To further understand the evolution of WOX genes, phylogenetic analysis of 103 WOXs (AtWOXs, PotriWOXs, OsWOXs, ZmWOXs, BradiWOXs, and PheWOXs) was carried out (Figure 2). The polypeptides used to construct the phylogenetic tree are shown in Supplementary Dataset S1. The maximum likelihood tree of the WOX family showed topologies similar to the neighbor-joining tree (Supplementary Figure S1). The WOX13 subfamily was considered an outgroup [40,41]. These WOXs divided into three distinct clades, as described before, namely, the ancient clade, intermediate clade, and WUS clade [8]. Each clade was well-supported by high bootstrap values, except for the ancient clade, which was consistent with previous studies $[9,42,43]$. To further investigate the evolutionary relationship of the WOX family, nine sub-groups were identified based on their phylogenetic topology, namely, WOX10/13/14 of the ancient clade, WOX8/9 and WOX11/12 of the intermediate clade, and WUS, WOX2, WOX3, WOX5/7, WOX4, and WOX1/6 of the WUS clade (Figure 2). This is consistent with previous studies [42].

WOXs underwent gene expansion in both dicots and monocots, particularly for the WUS and intermediate clades. The WUS and intermediate clades contained more genes than the ancient clade (Table 1). The evolutionary topologies of the sub-groups were clearly separated between dicots and monocots, and the topologies of WOX10/13/14 between dicots and monocots were hierarchical. Members of WOX11/12, WUS, WOX2, WOX3, WOX5/7, and WOX4 formed two separate branches of monocots and dicots with higher support values. This suggests that the genes of WOX10/13/14 occurred before the divergence of monocots and dicots and varied only slightly as the categories differentiated. The ancient members of WOX11/12, WUS, WOX2, WOX3, WOX5/7, and WOX4 also existed before the divergence of monocots and dicots, and diversified with different evolutionary rates after their divergence [44].

\subsection{Specific Domain Evolution of WOXs from Different Plants}

The functional domains of transcription factors always appear to diverge at reduced rates because of their critical role in protein function [45]. The HD is the basic feature of HB TFs and originated before the divergence of eukaryotes [7,8]. Some conserved amino acid sites of the HD were identified among plants (Figure 2), which might limit the variation of WOXs. Although the HD is conserved, the position of the WOX proteins of Chlorophyta and higher plants is different. In Chlorophyta, the HD is located closer to the C-terminal of proteins, while the HD of higher plant is far from the C-terminal due to the new conserved domains formed in the C-terminal (Figure 2). The HDs of GRMZM2G010929-P02 and PH02Gene09575.t2 are also located near the C-terminal (Figure 2). These two genes were clustered in WOX11/12 and WUS with low support.

Besides conserved HDs, other conserved domains of WOXs formed during the expansion of the WOX family. WOXs of each clade contain one or more specific domains (Figure 2). Although genes of the ancient clade divided into sub-branches close to Chlorophyta, the WOXs of dicots and monocots were in highly segregated clusters in the ancient clade. Motif 4, around 40 amino acid residues long, was found among the WOX10/13/14 of dicots and monocots. The homologous genes of AtWOX10 and AtWOX14 were not identified in other species. The specific domains of AtWOX10 and AtWOX14 are similar to Micpus-48125 of Micromonas pusilla, Micsp-RCC299 of Micromonas sp., and Ostlu-27102 of Ostreococcus tauri, which contain HDs and Motif 4 (Figure 2). Motif 5 was discovered in WOX13s, but not in WOX10/14s. Motif 6 was only found in the C-terminal of the WOX13s of Oryza sativa, Zea mays, Brachypodium distachyon, and bamboo, but not in Populus trichocarpa and Arabidopsis thaliana However, certain conserved amino acids of Motif 6 were found in the same position of AtWOX13 and PotriWOX13s (Supplementary Figure S2). We speculate that the origins of Motifs 5 and 6 are the same in dicots and monocots, but varied at different evolutionary rates.

The intermediate clade included WOX8/9s and WOX11/12s. Although Micpus-54493 clustered in the branch sister to intermediate clade, the bootstrap value was quite low (Figure 2). The topological structure of the intermediate clade divided into four sub-branches based on evolution, consisting of 
WOX8/9 of monocots, WOX8/9 of dicots, WOX11/12 of monocots, and WOX11/12 of dicots (Figure 2). Four motifs were found in the intermediate clade. Motif 7 was found at the N-terminal of most WOX8/9 proteins, which was not existent in the WOX11/12 proteins (Figure 2). Motifs 8 and 9 were located at the C-terminal of some WOX8/9s and all WOX11/12s. Moreover, AtWOX9 contained low-resolution Motif 10 in the same position as WOX11/12s (Figure 2). The loss/appearance of these domains might be associated with the functional differentiation of WOX8/9 and WOX11/12 in dicots and monocots.

The WUS clade included more genes than the other two clades. There were only two conserved motifs identified in the WUS clade, namely, the HD near the N-terminal and Motif 11 located at the C-terminal. Motif 11, containing the amino acid residue TLXLFP, is also called the WUS box and has been proven to be an important domain for repressor activity of the WUS clade proteins [46]. The WUS box has played an important role in the conserved function of the WUS clade. Indeed, the evolution of the WUS clade might be tied to the function of Motif 11. We could not find the WUS box in PheWUS, which might have a different function in P. edulis compared to other species.

\subsection{Evolution of WOXs in Bambusoideae with Different Ploidies}

Bamboos have diverse polyploidies. The evolution of Bambusoideae is driven by polyploidization [1]. The absolute copy number of WOXs increased in polyploid bamboo species compared with diploid species. The unrooted phylogenetic tree was constructed using 91 Bambusoideae WOX coding sequence (CDS) with the assessment of bootstrap value. The phylogenetic tree showed that WOXs of Bambusoideae were also classified into the ancient clade, intermediate clade, and WUS clade, with a high support value of over $80 \%$. Specific motif domains and gene structures were also identified (Figure 3). Three WOX-like genes (BamWOX8like, BamWOX9like, and PheWUSlike) of the 91 Bambusoideae were found to encode proteins with no HD (Figure 3), whereas 12 had partial HDs of motifs 2 and 3 at their N-terminal (Figure 3).

From the specific domain distribution observed, WOX13s of Bambusoideae were highly conserved, which was reflected not only in the protein length, but also in the domain position. However, WOX13s of Bambusoideae showed diverse numbers and lengths of exons and introns (Figure 3). Two groups-WOX4s and WOX5s - of Bambusoideae had high similarity at the protein level, and were also significantly more conserved at the genome level in different polyploidies (Supplementary Figures S3 and S4). These findings might be related to the important and conserved function of WOX4s, WOX5s, and WOX13s in Bambusoideae. 


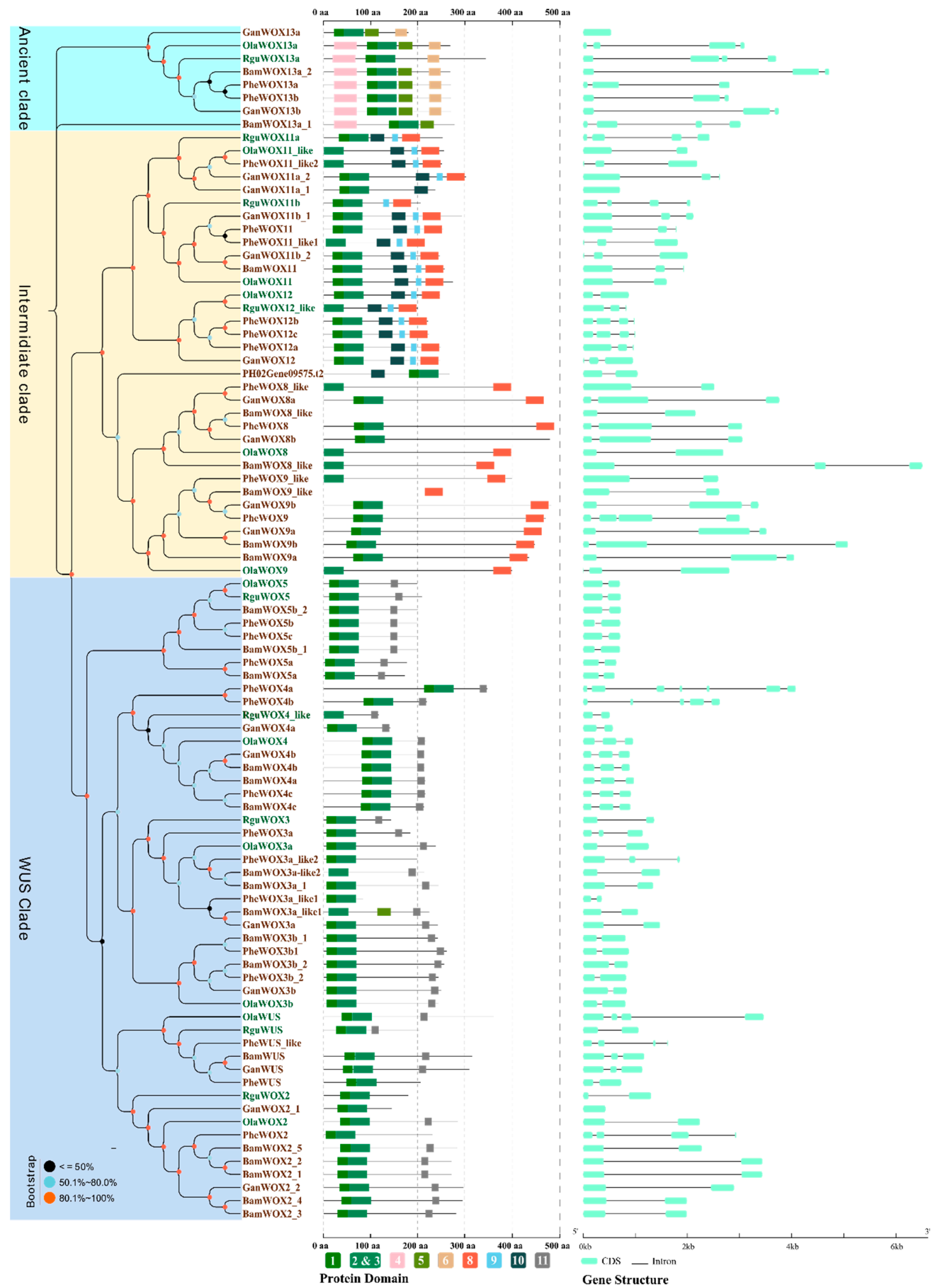

Figure 3. The phylogenetic tree of Bambusoideae WOXs. The neighbor-joining tree was constructed by the full-length CDS of Bambusoideae WOXs with bootstrap assessment of 1000 replicates. Each clade was assigned a different colored background. Green leaves of the phylogenetic tree stand for WOXs of herbaceous bamboos. Brown leaves of the phylogenetic tree stand for WOXs of woody bamboos. The protein domains and gene structures are drawn following each WOX. The name of each domain is the same as in Figure 1. 


\subsection{The Selective Constraints of Homologous Genes within and between Species}

To investigate the evolutionary rate of WOX genes, the $\mathrm{Ka} / \mathrm{Ks}$ ratios of sub-groups within and between species were calculated. A ratio of $\mathrm{Ka} / \mathrm{Ks}>1$ indicates accelerated evolution with positive selection, a ratio $=1$ indicates neutral selection, and a ratio $<1$ indicates negative/purifying selection. This analysis showed that the WOX family had strong purifying selection pressure, as almost all of $\mathrm{Ka} / \mathrm{Ks}$ ratios of the homologous WOXs detected were $<1$ (Supplementary Table S3).

Considering that strong purifying selection may shield positive selection at particular amino acid sites or regions, a sliding window analysis was performed to calculate the evolutionary rate for each codon. Most of the WOX13s pairs have largely encountered an intense purifying selection (average $\mathrm{Ka} / \mathrm{Ks}=0.15) . \mathrm{Ka} / \mathrm{Ks}$ ratios across the coding regions of OsWOX13_PheWOX13s and BradiWOX13_PheWOX13s were $<0.4$ (Figure $4 a, b$ ), indicating an intense purifying selection across all of the motif positions of WOX13. For gene pairs within species, the majority of the Ka/Ks ratios across the coding regions of PheWOX13a/b, GRMZMWOX13a/b, and GRMZMWOX13a/c were <1, except for the Motif 6 region (Figure 4c,d). A similar status was found at the HD region of PotriWOX13a_PotriWOX13b (Figure 4e). Motif 6 of ZmWOX13 and PheWOX13 and the HD of PotriWOX13s might undergo positive selection due to potential redundancy of paralogous function.
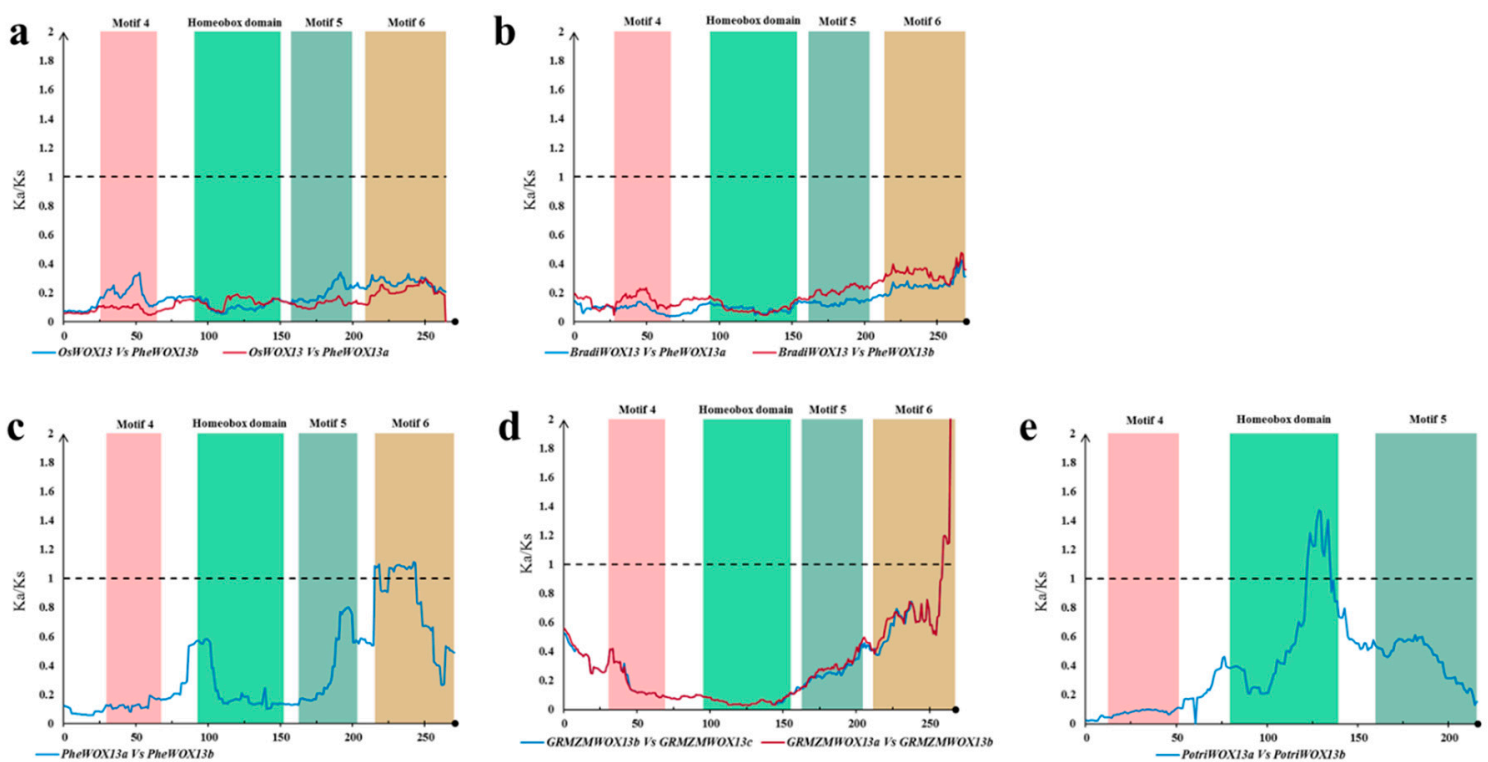

Figure 4. Sliding window of the WOX13 genes of the ancient clade. (a) The sliding window of orthologous WOX13s between P. edulis and Oryza sativa. (b) The sliding window of orthologous WOX13s between P. edulis and Brachypodium distachyon. (c-e) The sliding window of paralogous WOX13s of P. edulis, Zea mays, and Populus trichocarpa, respectively.

For the intermediate clade, several sliding window analyses of WOX8/9s and WOX11/12s were performed (Figure 5). Except for the coding region of Motif 8 of GRMZMWOX11a/b, it was obvious that the coding regions of Motif 7, the HD, and Motifs 8-10 went through an intensive purifying selection within the intermediate clade (Figure 5 and Supplementary Figure S5). Positive selection was found in the region between the HD and Motif 8 of GRMZMWOX8a/b (Figure 5a). A similar trace could be found in the WOX8/9 pairs in Arabidopsis thaliana, Oryza sativa, Zea mays, and Brachypodium distachyon (Figure 5b), and in the WOX8 pairs between different species (Figure $5 c$ ). The results show a positive selection in the non-conserved region near Motif 8 . The coding region upstream of Motif 8 of WOX8/9 might generate some new function motifs. Particularly, Motif 10, purported to be the specific domain of WOX11/12, was detected with low distinguishability (data not shown) in AtWOX9, while not found in AtWOX8. The alignment region of Motif 10 of AtWOX9/8 underwent positive selection (Figure 5b). 

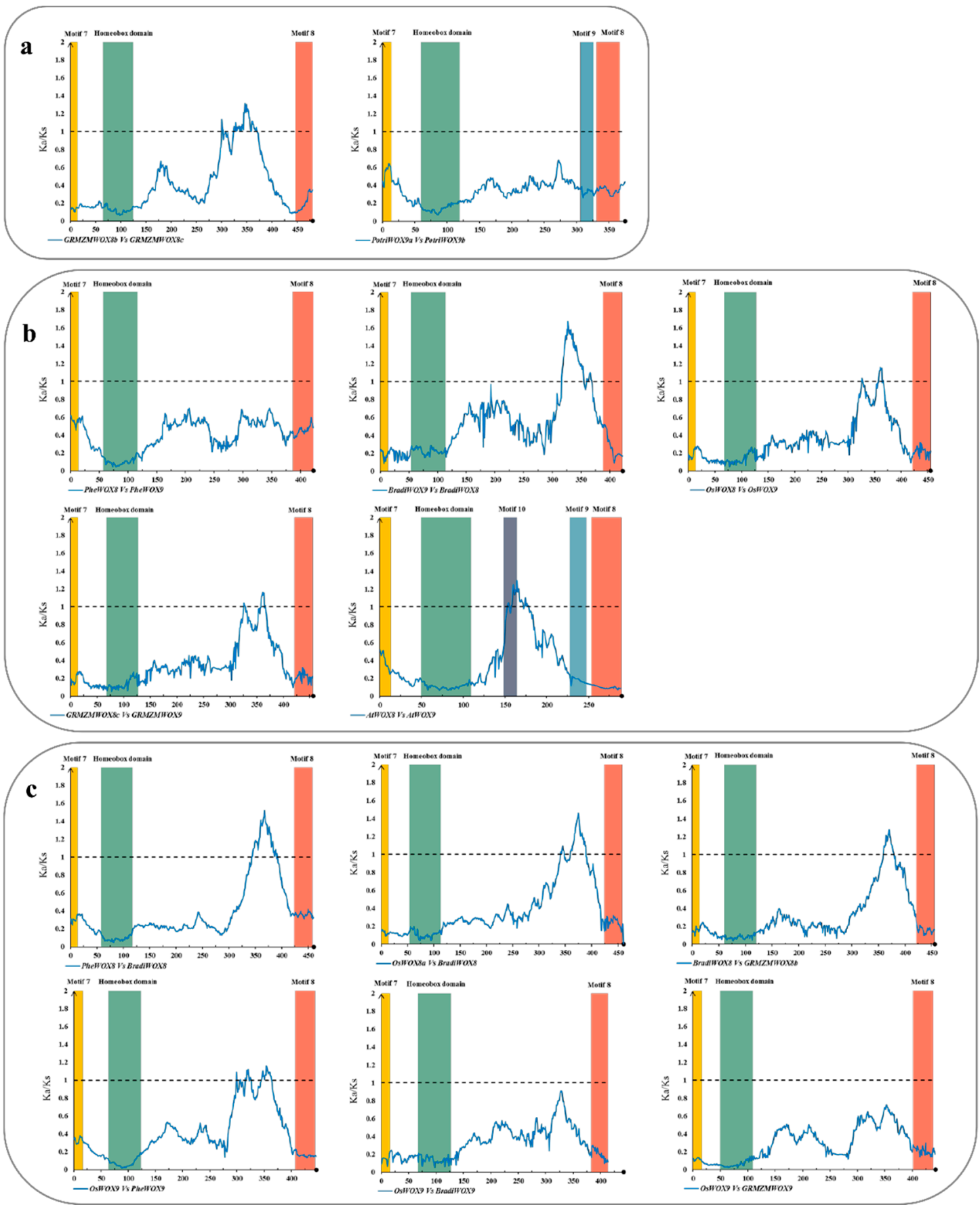

Figure 5. Sliding window of the WOX8/9 genes of the intermediate clade. (a) The sliding window of paralogous genes of WOX8/9. (b) The sliding window of orthologous genes of WOX8s. (c) The sliding window of orthologous genes of WOX9s.

Members of the WUS clade function as repressors due to the presence of the WUS box. Similar to the specific domain found in other clades, strong purifying selection was found at the region of the HD and the WUS box between/within sub-groups within species, suggesting the importance of the HD and the WUS box for the WUS clade. Positive selection pressure affected the regions between the HD and the WUS box within species, such as PheWOX2/3a, PheWOX3a/b, PheWOX4a/b, PotriWOX2a/b, GRMZMWOX3a/b, PotriWOX1a/b, and AtWOX1/6 (Supplementary Figure S6). Sequence variation was also found outside of the region between the HD and the WUS box in the orthologous genes of different species, such as OsWOX2_GRMZMWOX2, BradiWOX2_GRMZMWOX2, GRMZMWOX3a_PheWOX3a, 
and AtWOX3_OsWOX3b (Supplementary Figure S7). These findings evidence the diverse rates of generation of potential functions for members of the WUS clades.

\subsection{The Expansion of WOXs in Bambusoideae}

The majority of Bambusoideae WOXs underwent strong purifying selection pressure during polyploidization (Supplementary Table S4). Ks value can be used to indicate the divergence time of the duplication event [1,2]. In diploid herbaceous species (O. latifolia and R. guianensis), two pairs of WOX duplications, namely OlaWOX3a-OlaWOX3b and RguWOX11a-RguWOX11b, were found with higher Ks versus the majority of WOX duplications in polyploid woody bamboo species (B. amplexicaulis, G. angustifolia, and P. edulis) (Figure 6). OlaWOX3a-OlaWOX3b and RguWOX11a-RguWOX11b may have occurred by segmental duplications before bamboo speciation. In B. amplexicaulis, G. angustifolia, and P. edulis, the WOX3a-WOX3b pair also showed the high Ks similarity with O. latifolia (Figure 6), indicating the divergence of WOX3a-WOX3b likely was the result of segmental duplications before the divergence of herbaceous and woody bamboo. In tetraploid woody bamboo species, the duplication pairs of GanWOX13a-GanWOX13b, GanWOX11a-GanWOX11b, PheWOX11-PheWOX11_like2, GanWOX8a-GanWOX8b, PheWOX8-PheWOX8_like, GanWOX9a-GanWOX9b, PheWOX5a-PheWOX5b/c, GanWOX4a-GanWOX4b, and PheWOX4a-PheWOX4c, that were not tightly cluster in the tree, showed a Ks distance of 0.24 0.49 which indicated a divergence times of 18.4 37.7 Mya (Table S4). The polyploidization events occured 19.7 22.0 Mya which gave rise to the three major lineages, a hexaploid (AABBCC) and two tetraploids (BBCC and CCDD) [1]. These WOXs expansion in polyploidy might have resulted from an allopolyploid hybridization. In contrast, the gene pair of PheWOX13a/b, PheWOX11-PheWOX11-like1, PheWOX12b/c, PheWOX5b/c, and PheWOX3b1/b2 were cluster tightly, and showed a divergence times of 2.4 11.9 Mya (Table S4), which very likely resulted from the segmental DNA duplications. In B. amplexicaulis, more duplications of WOX2 were found with a narrow Ks distance of 0.02-0.21, suggesting frequent segmental duplications of WOX2 in recent times.

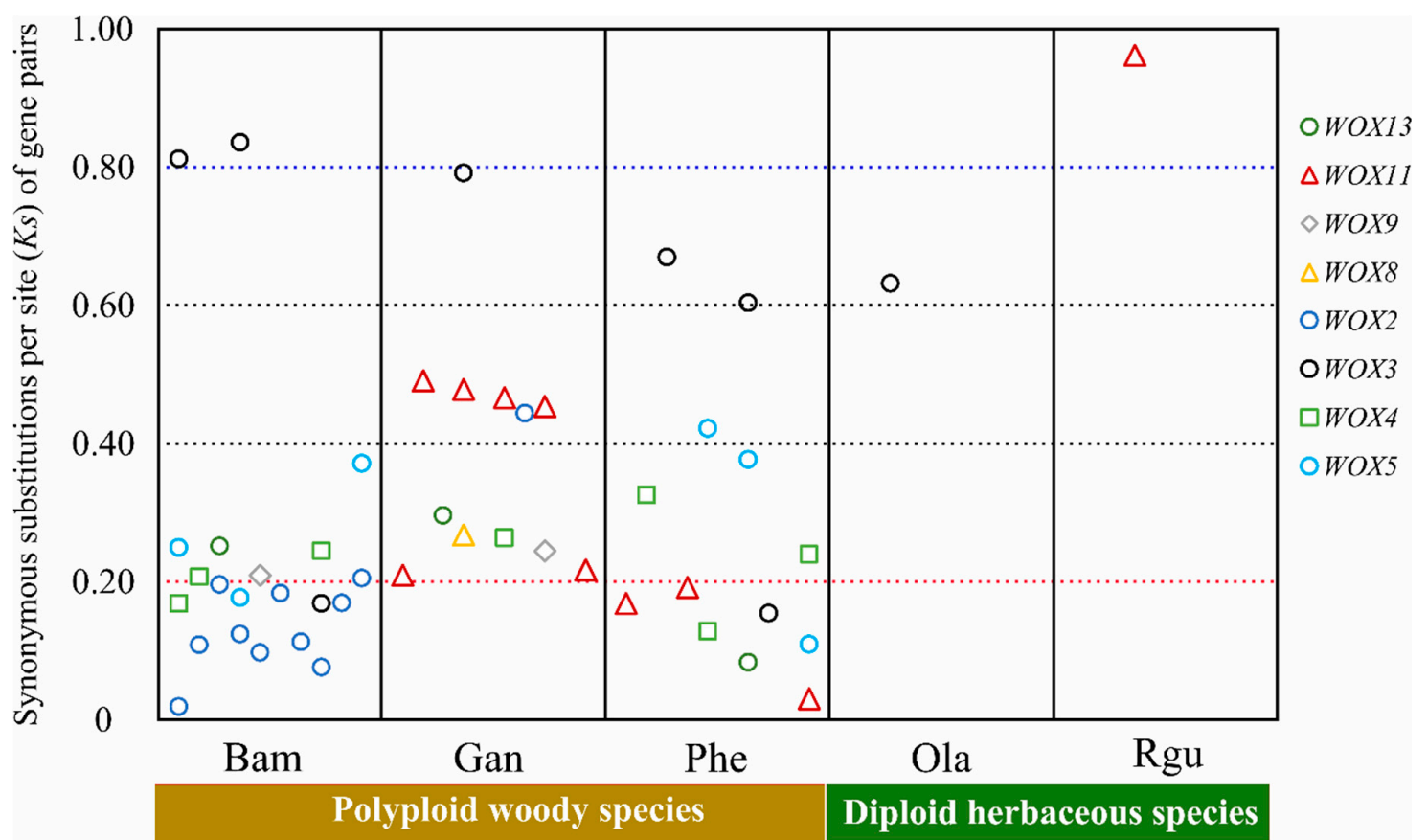

Figure 6. The duplications of the Bambusoideae WOXs of herbaceous and woody bamboos. The colored symbols represent different members of the WOX family. Each symbol indicates a homologous gene pair on the Ks distribution plot. The $\mathrm{y}$-axis indicates the calculated Ks value of each gene pair. 


\subsection{The Similar cis-Element Positions of the Orthologous WOX Promoters in Bambusoideae}

To evaluate potential upstream regions for potential common cis-regulatory sequences, we scanned regions 1500 bp upstream of the start codons for TF-binding motifs using PlantRegMap. TF-binding sites in the WOX promoters were identified based on the TF-binding motif dataset of PlantRegMap (Supplementary Table S5). The promoters of BamWOX4a/b/c, PheWOX4c, GanWOX4b, and OlaWOX4 were clustered in same branch (Supplementary Figure S7). MP00302/MP00227 was found generally at around the $-100 \mathrm{bp}$ position of these promoters (Figure 7a), but not found in the promoters of OsWOX4 or AtWOX4 (Supplementary Table S5). The B3 protein binding element, MP00083, was found in a similar region as that of the promoters of BamWOX $4 a / c$ and PheWOX4b/c (Figure 7a, Supplementary Table S5). MP00265 (CA[T/A]TCA[T/A]TCA), recognized by WUS, was found in the promoters of PheWOX4b (-970 -959 bp) and OsWOX4 (-1278 -1268 bp), while not found in the promoters of AtWOX4 or the other bamboo WOX4s (Supplementary Table S5). The promoters of PheWOX5b/c, BamWOX5b1/b2, RguWOX5, and OlaWOX5 clustered in same branch (Supplementary Figure S7). Two conserved positions were found in these promoters, namely, $-400 \sim-300 \mathrm{bp}$ and $-900 \sim-800 \mathrm{bp}$ upstream from the initiation codon (Figure 7b). At the position of $-400 \sim-300 \mathrm{bp}$, two consistent cis-elements, namely, MP00407/MP00580 and MP00253/MP00540, were found. MP00407 was also found in the promoters of AtWOX5 and OsWOX5. MP00114/MP00134 was scanned at the position of -900 -800 bp. The Bambusoideae WUS and WOX3a/b were clustered in three separate branches with high support (Supplementary Figure S7). A GA-rich element (MP00253/MP00540) was also found conserved in the promoters of bamboo and rice WOX3/WUS (Figure 7c). A MADS-binding sites (MP00076) was found in most WOX3 and WUS promoters and was predicted to be the target of PH02Gene23951.t1, homology to OsMADS56 of rice (Figure 7c). OsMADS56 regulates rice flowering antagonistic with OsMADS50 [47]. The promoters of BamWOX8, GanWOX8a, and OlaWOX8 showed two similar cis-elements, namely, MP00411/MP00302/MP00227 and MP00513/MP00249 (Supplementary Figure S8a). MP00076 was found at the promoters of BamWOX9b, GanWOX9b, OlaWOX9, and PheWOX9. An MYB-binding site (MP00513/MP00249) was also found in these promoters, 70 bp away from MP00076. MP00513/MP00249 was also found in the promoters of AtWOX9 and OsWOX9. At 1000 bp upstream from the start codon, abundant AP2/ERF-binding sites were found in the BamWOX9b, OlaWOX9, and PheWOX9 promoters (Supplementary Figure S8b). These sites, found approximately positioned in the promoters of bamboos' orthologous WOXs, may play an important role in regulating the expression of WOXs. 


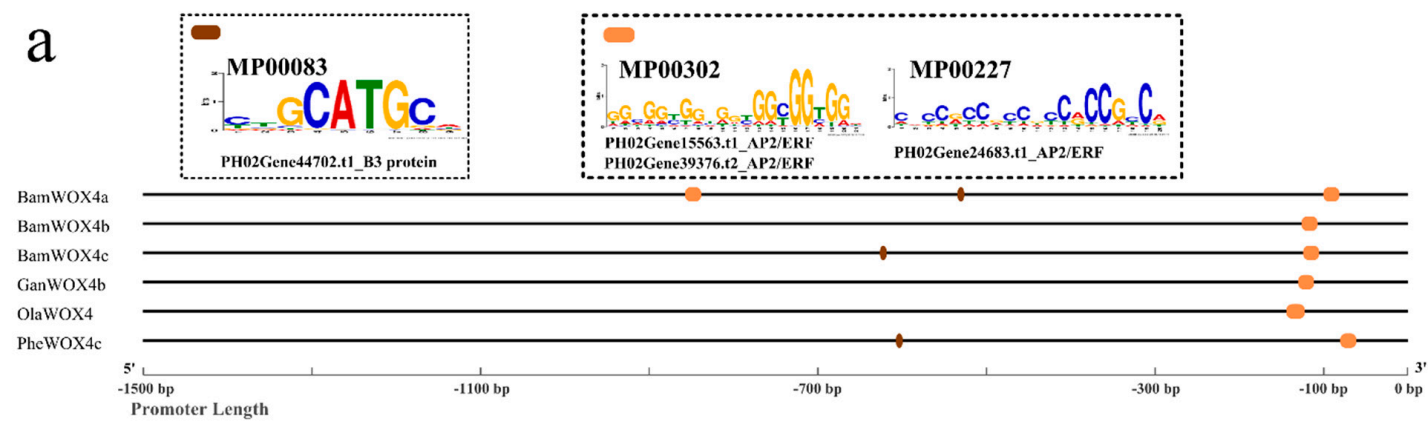

b
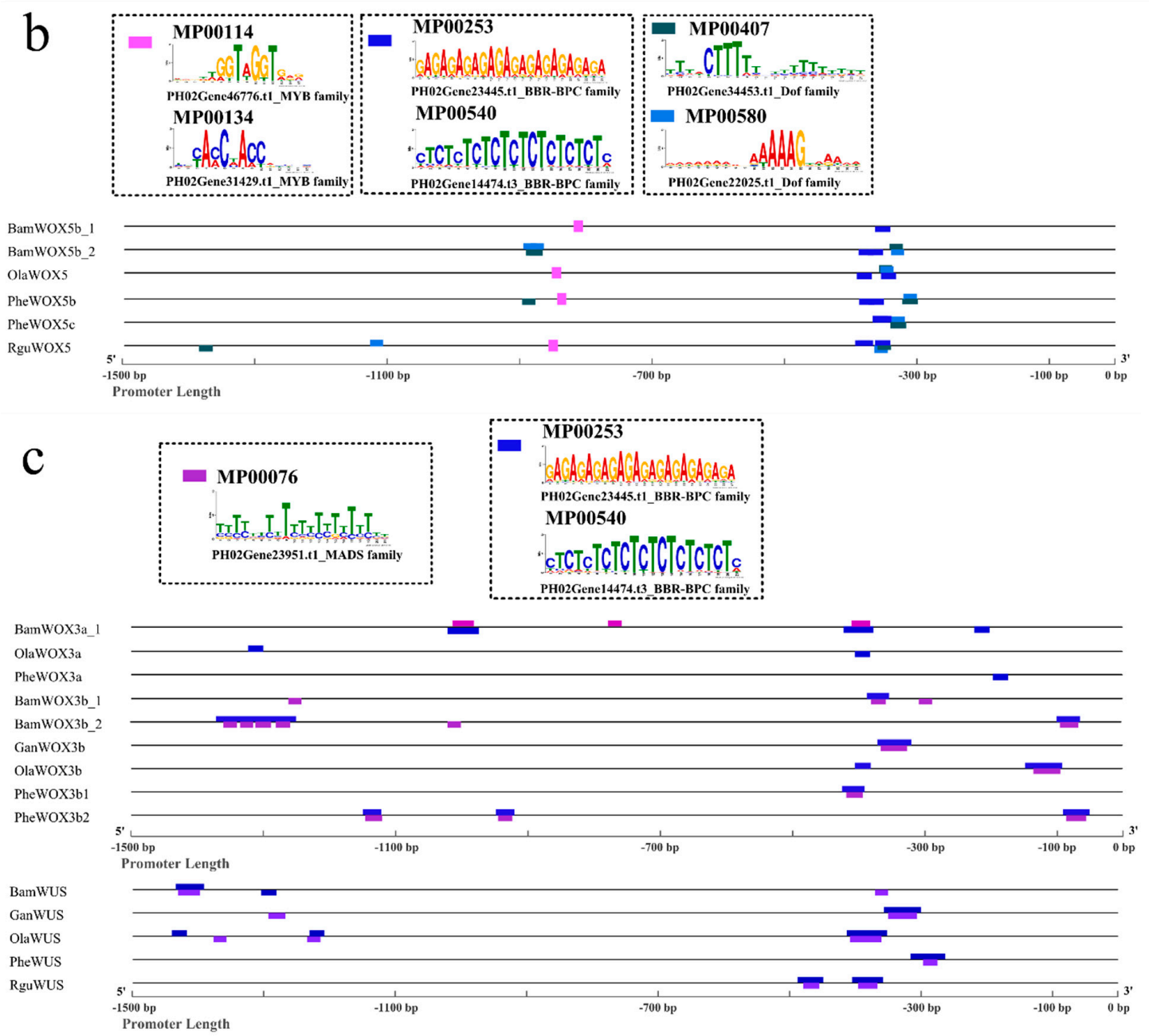

Figure 7. The conserved cis-element distribution in the promoters of Bambusoideae WOX4 (a), WOX5 (b), and WOX3 and WUS (c). The 1500-bp-long promoters were analyzed by PlantRegMap. The ID of the cis-elements was in accordance with the transcription factor (TF) binding motif dataset of PlantTFDB and is shown with different colored rectangles. The TFs were predicted by comparing homologous genes with TF datasets of Arabidopsis (http://planttfdb.cbi.pku.edu.cn/index.php?sp=Ath).

\section{Discussion}

\subsection{Conserved Domains were Retained during Species Differentiation}

The evolution of the WOX family played pivotal roles in morphological innovations during the evolutionary history of species differentiation in the plant kingdom [8,43,44,48-52]. The large-scale identification of the WOX family from Viridiplantae has been extensively studied, including species of lower and higher plants [42]. The ancient clade comprises WOX10/13/14 proteins from charophytes, 
bryophytes, gymnosperms, lycophytes, ferns, and angiosperms. The intermediate clade contains WOX8/9 and WOX 11/12 proteins from gymnosperms and angiosperms. The WUS clade includes WUS, WOX5/7, WOX3, WOX1/6, WOX4, and WOX2 proteins from gymnosperms and angiosperms, and some other WOX proteins of lycophytes and ferns that cluster into the branch that separates the subclades of gymnosperms and angiosperms [42]. It is clear that WOXs are ubiquitous in most plants, except for rhodophytes, and that members of the major clades were formed alongside plant evolution.

In addition to HDs, which are present as DNA-binding domains in all of the WOX members $[9,40,42,53]$, ten specific domains of three major WOX clades were identified in this study. Similar positions and high conservatism could be observed from the evolutionary topology (Figure 2). Motif 4 is found in the N-terminal domain of most WOX proteins in the ancient clade [9,40,42]. Motif 5 is found in the C-terminal of most WOX13 proteins, but absent in WOX10/14 proteins [9]. Moreover, a new specific domain, Motif 6, was identified in the C-terminal of WOX13 in rice, maize, Brachypodium distachyon, and five bamboo species (Figures 2 and 3). A species-wide analysis of Motif 6 was performed in the WOX13s of monocots and dicots, where Motif 6 was found in the WOX13s of $86.8 \%$ of monocots (33 of 38 monocots) and 32.0\% dicots (32 of 100 eudicots, in which 24 species belong to asterids) (data not shown). This suggests that the conservation of Motif 6 shows species specificity. Motifs 8-9 (named T2WOX) have been reported as specific domains of the intermediate clade in research of the WOX family from 267 species [42]. However, Motif 9 cannot be observed in WOX8/9s of rice, maize, Brachypodium distachyon, or five bamboo species. As per previous reports, Motif 10 is a typical domain found in WOX11/12s [9]. Motif 10 (the WUS box) can be found in the majority of the members of the WUS clade $[9,12,42,46,54,55]$. Moreover, an EAR-like motif, $\mathrm{L}(\mathrm{D} / \mathrm{E}) \mathrm{L}(\mathrm{R} / \mathrm{S}) \mathrm{L}$, was found in WUS and WOX5/7 proteins [9,42], but not found in the other subclade of the WUS clade.

\subsection{The Evolution of the WUS Clade was Likely Selected by its Conserved and Essential Function in Plants}

The WOX family regulates key plant developmental processes, especially for organ morphogenesis. WOXs have essential functions in cell population identification and differentiation, in regulating stem cell identity in SAM and root apical meristems (RAM) and vascular cambium [11,12,56-58], in polarity pattern formation [21], and in the development of lateral organs [59,60]. Members of the WOX family are highly conserved, indicating their similar functions among species, which is reflected in the conserved motif found in each clade among the different species.

The functions of AtWUS and AtWOX5 are complementary to each other in the SAM and RAM of Arabidopsis [12,57] via a similar regulative signaling pathway [56,61,62]. Null mutations of Arabidopsis PRESSED FLOWER1 (PRS1, AtWOX3) prevent the initialization of lateral organs and cause the preprimordial deletion of lateral stipules from vegetative leaves and lateral sepals/stamens of flowers [51], which can be rescued by AtWUS and AtWOX4 when driven by the PRS1 native promoter [19,55]. Null mutations of WOX3s cause narrow leaf phenotypes in some monocots, like maize [49], rice [63], and barley [20], which are similar to the WOX1 mutants of some dicots, like Petunia (maw) [60], Medicago truncatula (stf) [64], and Nicotiana sylvestris (lam1) [65]. All members of the Arabidopsis WUS clade, except for WOX7 (loss of the WUS box, Figure 2), could complement lam 1 mutation when driven by the stf native promoter of Medicago truncatula [46]. The members of the intermediate and ancient clades driven by the stf promoter cannot complement lam 1 mutation unless a supererogatory repressor domain adheres [46], indicating the importance of the WUS box as transcriptional repressor domain. Intensive purifying selection was found in the coding regions of both the HD and the WUS box domains among species by sliding window analysis (Supplementary Figures S6 and S7), confirming the importance of the presence of the HD and the WUS box for the conserved function of the WUS clade. WOX genes of WUS clade are functionally redundant, and could replace each other if expressed in the necessary developmental time and space. It seems that the subfunctionalization of the WUS clade genes most likely depends on the diverse promoter specificity of paralogous genes. In bamboos, conserved evolution of Bambusoideae WOX4s and WOX5s was 
found at the transcriptional and translational levels, as well as cis-element distribution in the promoter region, which indicates the important function of WOX4s and WOX5s for bamboo development.

\subsection{The Conserved cis-Element Distribution of Bambusoideae WOXs May Relate to Their Functions during Development}

In our study, some particular cis-elements found at the same positions of orthologous genes of different bamboos may regulate bamboo development indispensability. A WUS binding site was found in the promoters of PheWOX4a and OsWOX4. In rice, OsWUS was expressed in the premeristem zone in the early stage, and then disappeared in the axillary meristem after its establishment. However, OsWOX4 was expressed in the established axillary meristem, but either no or very weak expression was detected in the early premeristem zone [17]. MP00265 might be related to the switch between OsWUS and OsWOX4 found during axillary formation in rice.

MP00253/MP00540 were found at the same positions of the WOX5, WOX3, and WUS promoters, which are GA-rich elements (GAGA-repeats) present in the regulatory sequences of genes involved in developmental processes [66,67]. MP00407/MP00580, found in the WOX5 promoter, showed a core sequence of 5'-AA[AG]G-3' recognized by two PheDofs (PH02Gene34453.t1 and PH02Gene22025.t1), homologous to AtDOF5.6 (also named as HCA2) and AtDOF3.6 (also named as OBP3) of Arabidopsis thaliana. Two positions of MP00407 were found at the regions of -613 -593 bp and -967 -947 bp in the promoter of AtWOX5, and found at the position of $-927 \sim-907 \mathrm{bp}$ in the promoter of OsWOX5 (Supplementary Table S5). HCA2 expressed in the vascular tissues and pericycle of primary roots has played a vital role in the promotion of both cambium activity and phloem specification [68]. OBP3 has been reported as a salicylic acid-induced gene predominantly expressed in roots [69], and overexpression of OBP3 results in defective roots [70]. Another site in the WOX5 promoter, MP00114/MP00134, was recognized by PH02Gene31429.t1 and PH02Gene46776.t1, homologous to AT1G09540.1 (AtMYB61) and AT3G61250.1 (AtMYB17, LMI2), respectively. AtMYB61 coordinates the target genes required for xylem formation, xylem cell differentiation, lateral root formation, etc. [71]. AtMYB17 mainly functions in the transition of meristem identity from vegetative growth to flowering [72]. The $-400 \mathrm{bp}-300 \mathrm{bp}$ and $-900 \sim-800 \mathrm{bp}$ regions of the bamboo WOX5 promoter may play a vital role in regulating the expression of WOX5 during bamboo adventitious root formation.

MP00076 is found in the majority of bamboo WOX3 and WUS promoters. We investigated the AtWOX3 promoter, and also found MP00076 in the region of -662 to $-638 \mathrm{bp}$ and -1038 to $-1018 \mathrm{bp}$. AtSOC1 (AT2G45660.1) could bind to MP00076, and promote inflorescence meristem identity during the transition to flowering of Arabidopsis thaliana [73]. MP00076 was also found in the promoters of BamWOX9b, GanWOX9b, OlaWOX9, and PheWOX9. WOX3, WUS, and WOX9 have been observed to regulate floral development [74]. We speculate that bamboo WUS, WOX3, and WOX9 may mediate meristem floral development and architecture, similar to previous studies.

\section{Conclusions}

In this study, 12 OlaWOXs, 9 RguWOX, 18 GanWOXs, 27 PheWOXs, and 26 BamWOXs were identified and categorized into ancient, intermediate, and WUS clades. Except for a universal HD found in all WOXs, eight specific domains were identified-three found in the ancient clade, four found in the intermediate clade, and one (the WUS box) found in the WUS clade. Intensive purifying selection in the coding region of specific domains leads to the conserved function of orthologous genes. For Bambusoideae WOXs, polyploidy is the major driver of the expansion of the WOX family. A low evolutionary rate of orthologous genes was found within the WOX family of Bambusoideae. AP2/ERF protein-binding regions and $\mathrm{B} 3$ protein-binding regions were found at the similar position of bamboo WOX4 promoters. Moreover, MYB protein-binding regions and Dof protein-binding regions were also discovered in the promoters of bamboo WOX5. These conserved cis-elements may play an important role in regulating the expression of WOX4 and WOX5. Flowering-related MADS binding sites were 
found in the promoters of bamboo WOX3, WUS, and WOX9, which indicate their potential roles in regulating bamboo flower formation.

Supplementary Materials: The following are available online at http://www.mdpi.com/2218-273X/10/5/739/s1, Figure S1: The Maximum Likelihood tree of WOXs from dicot and monocot; Figure S2: The alignment of WOX13s amino acid sequences; Figure S3: The alignment of the genome sequences of Bambusoideae WOX4s; Figure S4: The alignment of the genome sequences of Bambusoideae WOX5s; Figure S5: Sliding-window of the WOX11/12s; Figure S6 Sliding-window of the paralogous genes in the WUS clade; Figure S7: Sliding-window of the orthologous genes in the WUS clade; Figure S8: The conservative cis-element distribution in the promoters of Bambusoideae WOX8(a), WOX9(b), Tables S1: The informations of Bambusoideae WOXs; Tables S2: The informations of dicot and monocot WOXs used in this study; Table S3: The Ka/Ks ratio of dicot and monocot WOXs in sub-group; Tables S4: The $\mathrm{Ka} / \mathrm{Ks}$ ratio of Bambusoideae WOXs in sub-group; Table S5: The cis-elements predicted in the promoter of Bambusoideae WOXs, Dataset S1: Polypeptides used to construct phylogenetic tree of AtWOXs, PotriWOXs, OsWOXs, ZmWOXs, BradiWOXs, and PheWOXs.

Author Contributions: X.L. and J.G. conceived and designed the experiments, and wrote the paper; X.L., J.L., collected and identified WOXs; Z.C., M.C., and X.L. performed visualization of the data. H.Z. and Z.C. edited the paper. All authors have read and agreed to the published version of the manuscript.

Funding: This research was funded by the National Key R\&D program of China (Grant No. 2018YFD0600100) and the Fundamental Research Funds of ICBR (Grant No. 1632018006). The authors wish to express their appreciation to the reviewers for this manuscript.

Conflicts of Interest: The authors declare no conflict of interest. The funding sponsors contributed reagents/ materials/analysis tools in this paper.

\section{References}

1. Guo, Z.H.; Ma, P.F.; Yang, G.Q.; Hu, J.Y.; Liu, Y.L.; Xia, E.H.; Zhong, M.C.; Zhao, L.; Sun, G.L.; Xu, Y.X.; et al. Genome sequences provide insights into the reticulate origin and unique traits of woody bamboos. Mol. Plant 2019, 12, 1353-1365. [CrossRef] [PubMed]

2. Peng, Z.; Lu, Y.; Li, L.; Zhao, Q.; Feng, Q.I.; Gao, Z.; Lu, H.; Hu, T.; Yao, N.; Liu, K.; et al. The draft genome of the fast-growing non-timber forest species moso bamboo (Phyllostachys heterocycla). Nat. Genet. 2013, 45, 456. [PubMed]

3. Janzen, D.H. Why bamboos wait so long to flower. Annu. Rev. Ecol. Syst. 1976, 7, 347-391. [CrossRef]

4. Wei, Q.; Jiao, C.; Guo, L.; Ding, Y.; Cao, J.; Feng, J.; Dong, X.; Mao, L.; Sun, H.; Yu, F. Exploring key cellular processes and candidate genes regulating the primary thickening growth of $\mathrm{M}$ oso underground shoots. New Phytol. 2017, 214, 81-96. [CrossRef]

5. Li, L.; Cheng, Z.; Ma, Y.; Bai, Q.; Li, X.; Cao, Z.; Wu, Z.; Gao, J. The association of hormone signalling genes, transcription and changes in shoot anatomy during moso bamboo growth. Plant Biotechnol. J. 2018, 16, 72-85. [CrossRef]

6. Gehring, W.J.; Qian, Y.Q.; Billeter, M.; Furukubo-Tokunaga, K.; Schier, A.F.; Resendez-Perez, D.; Affolter, M.; Otting, G.; Wüthrich, K. Homeodomain-DNA recognition. Cell 1994, 78, 211-223. [CrossRef]

7. Ariel, F.D.; Manavella, P.A.; Dezar, C.A.; Chan, R.L. The true story of the HD-Zip family. Trends Plant Sci. 2007, 12, 419-426. [CrossRef]

8. Van der Graaff, E.; Laux, T.; Rensing, S.A. The WUS homeobox-containing (WOX) protein family. Genome Biol. 2009, 10, 248. [CrossRef]

9. Zhang, X.; Zong, J.; Liu, J.; Yin, J.; Zhang, D. Genome-wide analysis of WOX gene family in rice, sorghum, maize, Arabidopsis and poplar. J. Integr. Plant Biol. 2010, 52, 1016-1026. [CrossRef]

10. Haecker, A.; Groß-Hardt, R.; Geiges, B.; Sarkar, A.; Breuninger, H.; Herrmann, M.; Laux, T. Expression dynamics of WOX genes mark cell fate decisions during early embryonic patterning in Arabidopsis thaliana. Development 2004, 131, 657-668. [CrossRef]

11. Hirakawa, Y.; Kondo, Y.; Fukuda, H. TDIF peptide signaling regulates vascular stem cell proliferation via the WOX4 homeobox gene in Arabidopsis. Plant Cell 2010, 22, 2618-2629. [CrossRef] [PubMed]

12. Zhang, Y.; Jiao, Y.; Jiao, H.; Zhao, H.; Zhu, Y.-X. Two-step functional innovation of the stem-cell factors WUS/WOX5 during plant evolution. Mol. Biol. Evol. 2016, 34, 640-653. [CrossRef] [PubMed]

13. Tanaka, W.; Hirano, H.Y. Antagonistic action of TILLERS ABSENT1 and FLORAL ORGAN NUMBER2 regulates stem cell maintenance during axillary meristem development in rice. New Phytol. 2020, 225.2, 974-984. [CrossRef] [PubMed] 
14. Brand, U.; Fletcher, J.C.; Hobe, M.; Meyerowitz, E.M.; Simon, R. Dependence of stem cell fate in Arabidopsis on a feedback loop regulated by CLV3 activity. Science 2000, 289, 617-619. [CrossRef] [PubMed]

15. Pi, L.; Aichinger, E.; van der Graaff, E.; Llavata-Peris, C.I.; Weijers, D.; Hennig, L.; Groot, E.; Laux, T. Organizer-derived WOX5 signal maintains root columella stem cells through chromatin-mediated repression of CDF4 expression. Dev. Cell 2015, 33, 576-588. [CrossRef] [PubMed]

16. Lu, Z.; Shao, G.; Xiong, J.; Jiao, Y.; Wang, J.; Liu, G.; Meng, X.; Liang, Y.; Xiong, G.; Wang, Y. MONOCULM 3, an ortholog of WUSCHEL in rice, is required for tiller bud formation. J. Genet. Genom. 2015, 42, 71-78. [CrossRef]

17. Tanaka, W.; Ohmori, Y.; Ushijima, T.; Matsusaka, H.; Matsushita, T.; Kumamaru, T.; Kawano, S.; Hirano, H.-Y. Axillary meristem formation in rice requires the WUSCHEL ortholog TILLERS ABSENT1. Plant Cell 2015, 27, 1173-1184. [CrossRef]

18. Ohmori, Y.; Tanaka, W.; Kojima, M.; Sakakibara, H.; Hirano, H.-Y. WUSCHEL-RELATED HOMEOBOX4 is involved in meristem maintenance and is negatively regulated by the CLE gene FCP1 in rice. Plant Cell 2013, 25, 229-241. [CrossRef]

19. Shimizu, R.; Ji, J.; Kelsey, E.; Ohtsu, K.; Schnable, P.S.; Scanlon, M.J. Tissue specificity and evolution of meristematic WOX3 function. Plant Physiol. 2009, 149, 841-850. [CrossRef]

20. Yoshikawa, T.; Tanaka, S.-Y.; Masumoto, Y.; Nobori, N.; Ishii, H.; Hibara, K.-I.; Itoh, J.-I.; Tanisaka, T.; Taketa, S. Barley NARROW LEAFED DWARF1 encoding a WUSCHEL-RELATED HOMEOBOX 3 (WOX3) regulates the marginal development of lateral organs. Breed. Sci. 2016, 66, 416-424. [CrossRef]

21. Ueda, M.; Zhang, Z.; Laux, T. Transcriptional activation of Arabidopsis axis patterning genes WOX8/9 links zygote polarity to embryo development. Dev. Cell 2011, 20, 264-270. [CrossRef] [PubMed]

22. Lie, C.; Kelsom, C.; Wu, X. WOX2 and STIMPY-LIKE/WOX8 promote cotyledon boundary formation in Arabidopsis. Plant J. 2012, 72, 674-682. [CrossRef]

23. Liu, J.; Sheng, L.; Xu, Y.; Li, J.; Yang, Z.; Huang, H.; Xu, L. WOX11 and 12 are involved in the first-step cell fate transition during de novo root organogenesis in Arabidopsis. Plant Cell 2014, 26, 1081-1093. [CrossRef] [PubMed]

24. Hu, X.; Xu, L. Transcription factors WOX11/12 directly activate WOX5/7 to promote root primordia initiation and organogenesis. Plant Physiol. 2016, 172, 2363-2373. [CrossRef]

25. Laux, T.; Mayer, K.; Berger, J.; Jurgens, G. The WUSCHEL gene is required for shoot and floral meristem integrity in Arabidopsis. Development 1996, 122, 87-96. [PubMed]

26. Somssich, M.; Je, B.I.; Simon, R.; Jackson, D. CLAVATA-WUSCHEL signaling in the shoot meristem. Development 2016, 143, 3238-3248. [CrossRef] [PubMed]

27. Jin, J.; Tian, F.; Yang, D.-C.; Meng, Y.-Q.; Kong, L.; Luo, J.; Gao, G. PlantTFDB 4.0: Toward a central hub for transcription factors and regulatory interactions in plants. Nucleic Acids Res. 2017, 45.D1, D1040-D1045. [CrossRef]

28. Zhao, H.; Gao, Z.; Wang, L.; Wang, J.; Wang, S.; Fei, B.; Chen, C.; Shi, C.; Liu, X.; Zhang, H. Chromosome-level reference genome and alternative splicing atlas of moso bamboo (Phyllostachys edulis). GigaScience 2018, 7, giy115. [CrossRef]

29. Marchler-Bauer, A.; Bo, Y.; Han, L.; He, J.; Lanczycki, C.J.; Lu, S.; Chitsaz, F.; Derbyshire, M.K.; Geer, R.C.; Gonzales, N.R. CDD/SPARCLE: Functional classification of proteins via subfamily domain architectures. Nucleic Acids Res. 2016, 45, D200-D203. [CrossRef]

30. Chen, C.; Xia, R.; Chen, H.; He, Y. TBtools, a Toolkit for Biologists integrating various biological data handling tools with a user-friendly interface. BioRxiv 2018. [CrossRef]

31. Thompson, J.D.; Gibson, T.J.; Plewniak, F.; Jeanmougin, F.; Higgins, D.G. The CLUSTAL_X windows interface: Flexible strategies for multiple sequence alignment aided by quality analysis tools. Nucleic Acids Res. 1997, 25, 4876-4882. [CrossRef] [PubMed]

32. Tamura, K.; Peterson, D.; Peterson, N.; Stecher, G.; Nei, M.; Kumar, S. MEGA5: Molecular evolutionary genetics analysis using maximum likelihood, evolutionary distance, and maximum parsimony methods. Mol. Biol. Evol. 2011, 28, 2731-2739. [CrossRef] [PubMed]

33. He, Z.; Zhang, H.; Gao, S.; Lercher, M.J.; Chen, W.-H.; Hu, S. Evolview v2: An online visualization and management tool for customized and annotated phylogenetic trees. Nucleic Acids Res. 2016, 44, W236-W241. [CrossRef] [PubMed] 
34. Yang, Z. PAML 4: Phylogenetic analysis by maximum likelihood. Mol. Biol. Evol. 2007, 24, 1586-1591. [CrossRef] [PubMed]

35. Liang, H.; Zhou, W.; Landweber, L.F. SWAKK: A web server for detecting positive selection in proteins using a sliding window substitution rate analysis. Nucleic Acids Res. 2016, 34 (Suppl_2), W382-W384. [CrossRef]

36. Lynch, M.; Conery, J.S. The evolutionary fate and consequences of duplicate genes. Science 2000, 290, 1151-1155. [CrossRef]

37. Tian, F.; Yang, D.-C.; Meng, Y.-Q.; Jin, J.; Gao, G. PlantRegMap: Charting functional regulatory maps in plants. Nucleic Acids Res. 2020, 48, D1104-D1113. [CrossRef]

38. Fernández-Mazuecos, M.; Glover, B.J. The evo-devo of plant speciation. Nat. Ecol. Evol. 2017, 1, 0110. [CrossRef]

39. Zhang, Y.; Wu, R.; Qin, G.; Chen, Z.; Gu, H.; Qu, L.J. Over-expression of WOX1 Leads to Defects in Meristem Development and Polyamine Homeostasis in Arabidopsis F. J. Integr. Plant Biol. 2011, 53, 493-506. [CrossRef]

40. Deveaux, Y.; Toffano-Nioche, C.; Claisse, G.; Thareau, V.; Morin, H.; Laufs, P.; Moreau, H.; Kreis, M.; Lecharny, A. Genes of the most conserved WOX clade in plants affect root and flower development in Arabidopsis. BMC Evol. Biol. 2008, 8, 291. [CrossRef]

41. Lian, G.; Ding, Z.; Wang, Q.; Zhang, D.; Xu, J. Origins and evolution of WUSCHEL-related homeobox protein family in plant kingdom. Sci. World J. 2014. [CrossRef] [PubMed]

42. Wu, C.-C.; Li, F.-W.; Kramer, E.M. Large-scale phylogenomic analysis suggests three ancient superclades of the WUSCHEL-RELATED HOMEOBOX transcription factor family in plants. PLoS ONE 2019, 1. [CrossRef] [PubMed]

43. Nardmann, J.; Reisewitz, P.; Werr, W. Discrete shoot and root stem cell-promoting WUS/WOX5 functions are an evolutionary innovation of angiosperms. Mol. Biol. Evol. 2009, 26, 1745-1755. [CrossRef] [PubMed]

44. Nardmann, J.; Werr, W. Symplesiomorphies in the WUSCHEL clade suggest that the last common ancestor of seed plants contained at least four independent stem cell niches. New Phytol. 2013, 199, 1081-1092. [CrossRef]

45. Liu, L.; White, M.J.; MacRae, T.H. Transcription factors and their genes in higher plants: Functional domains, evolution and regulation. Eur. J. Biochem. 1999, 262, 247-257. [CrossRef]

46. Lin, H.; Niu, L.; McHale, N.A.; Ohme-Takagi, M.; Mysore, K.S.; Tadege, M. Evolutionarily conserved repressive activity of WOX proteins mediates leaf blade outgrowth and floral organ development in plants. Proc. Natl. Acad. Sci. USA 2013, 110, 366-371. [CrossRef]

47. Ryu, C.H.; Lee, S.; Cho, L.H.; Kim, S.L.; Lee, Y.S.; Choi, S.C.; Jeong, H.J.; Yi, J.; Park, S.J.; Han, C.D. OsMADS50 and OsMADS56 function antagonistically in regulating long day (LD)-dependent flowering in rice. Plant Cell Environ. 2009, 32, 1412-1427. [CrossRef]

48. Harrison, C.J.; Morris, J.L. The origin and early evolution of vascular plant shoots and leaves. Philos. Trans. R. Soc. B Biol. Sci. 2018, 373, 20160496. [CrossRef]

49. Nardmann, J.; Werr, W. The shoot stem cell niche in angiosperms: Expression patterns of WUS orthologues in rice and maize imply major modifications in the course of mono-and dicot evolution. Mol. Biol. Evol. 2006, 23, 2492-2504. [CrossRef]

50. Nardmann, J.; Werr, W. The invention of WUS-like stem cell-promoting functions in plants predates leptosporangiate ferns. Plant Mol. Biol. 2012, 78, 123-134. [CrossRef]

51. Nardmann, J.; Ji, J.; Werr, W.; Scanlon, M.J. The maize duplicate genes narrow sheath1 and narrow sheath2 encode a conserved homeobox gene function in a lateral domain of shoot apical meristems. Development 2004, 131, 2827-2839. [CrossRef] [PubMed]

52. Burki, F.; Inagaki, Y.; Bråte, J.; Archibald, J.M.; Keeling, P.J.; Cavalier-Smith, T.; Sakaguchi, M.; Hashimoto, T.; Horak, A.; Kumar, S. Large-scale phylogenomic analyses reveal that two enigmatic protist lineages, Telonemia and Centroheliozoa, are related to photosynthetic chromalveolates. Genome Biol. Evol. 2009, 1, 231-238. [CrossRef] [PubMed]

53. Li, X.; Hamyat, M.; Liu, C.; Ahmad, S.; Gao, X.; Guo, C.; Wang, Y.; Guo, Y. Identification and characterization of the WOX family genes in five Solanaceae species reveal their conserved roles in peptide signaling. Genes 2018, 9, 260. [CrossRef] [PubMed]

54. Palovaara, J.; Hakman, I. WOX2 and polar auxin transport during spruce embryo pattern formation. Plant Signal. Behav. 2009, 4, 153-155. [CrossRef] 
55. Ji, J.; Shimizu, R.; Sinha, N.; Scanlon, M.J. Analyses of WOX4 transgenics provide further evidence for the evolution of the WOX gene family during the regulation of diverse stem cell functions. Plant Signal. Behav. 2010, 5, 916-920. [CrossRef]

56. Schoof, H.; Lenhard, M.; Haecker, A.; Mayer, K.F.; Jürgens, G.; Laux, T. The stem cell population of Arabidopsis shoot meristems is maintained by a regulatory loop between the CLAVATA and WUSCHEL genes. Cell 2000, 100, 635-644. [CrossRef]

57. Sarkar, A.K.; Luijten, M.; Miyashima, S.; Lenhard, M.; Hashimoto, T.; Nakajima, K.; Scheres, B.; Heidstra, R.; Laux, T. Conserved factors regulate signalling in Arabidopsis thaliana shoot and root stem cell organizers. Nature 2007, 446, 811. [CrossRef]

58. Ikeda, M.; Mitsuda, N.; Ohme-Takagi, M. Arabidopsis WUSCHEL is a bifunctional transcription factor that acts as a repressor in stem cell regulation and as an activator in floral patterning. Plant Cell 2009, 21, 3493-3505. [CrossRef]

59. Lenhard, M.; Bohnert, A.; Jürgens, G.; Laux, T. Termination of stem cell maintenance in Arabidopsis floral meristems by interactions between WUSCHEL and AGAMOUS. Cell 2001, 105, 805-814. [CrossRef]

60. Vandenbussche, M.; Horstman, A.; Zethof, J.; Koes, R.; Rijpkema, A.S.; Gerats, T. Differential recruitment of WOX transcription factors for lateral development and organ fusion in Petunia and Arabidopsis. Plant Cell 2009, 21, 2269-2283. [CrossRef]

61. Stahl, Y.; Wink, R.H.; Ingram, G.C.; Simon, R. A signaling module controlling the stem cell niche in Arabidopsis root meristems. Curr. Biol. 2009, 19, 909-914. [CrossRef] [PubMed]

62. Yadav, R.K.; Perales, M.; Gruel, J.; Girke, T.; Jönsson, H.; Reddy, G.V. WUSCHEL protein movement mediates stem cell homeostasis in the Arabidopsis shoot apex. Genes Dev. 2011, 25, 2025-2030. [CrossRef] [PubMed]

63. Cho, S.H.; Yoo, S.C.; Zhang, H.; Pandeya, D.; Koh, H.J.; Hwang, J.Y.; Kim, G.T.; Paek, N.C. The rice narrow leaf2 and narrow leaf3 loci encode WUSCHEL-related homeobox 3 A (O s WOX 3 A) and function in leaf, spikelet, tiller and lateral root development. New Phytol. 2013, 198, 1071-1084. [CrossRef] [PubMed]

64. Tadege, M.; Lin, H.; Bedair, M.; Berbel, A.; Wen, J.; Rojas, C.M.; Niu, L.; Tang, Y.; Sumner, L.; Ratet, P. STENOFOLIA regulates blade outgrowth and leaf vascular patterning in Medicago truncatula and Nicotiana sylvestris. Plant Cell 2011, 23, 2125-2142. [CrossRef] [PubMed]

65. McHale, N.A.; Marcotrigiano, M. LAM1 is required for dorsoventrality and lateral growth of the leaf blade in Nicotiana. Development 1998, 125, 4235-4243. [PubMed]

66. Hecker, A.; Brand, L.H.; Peter, S.; Simoncello, N.; Kilian, J.; Harter, K.; Gaudin, V.; Wanke, D. The Arabidopsis GAGA-binding factor basic pentacysteine6 recruits the polycomb-repressive complex1 component like heterochromatin protein1 to GAGA DNA motifs. Plant Physiol. 2015, 168, 1013-1024. [CrossRef]

67. Meister, R.J.; Williams, L.A.; Monfared, M.M.; Gallagher, T.L.; Kraft, E.A.; Nelson, C.G.; Gasser, C.S. Definition and interactions of a positive regulatory element of the Arabidopsis INNER NO OUTER promoter. Plant J. 2004, 37, 426-438. [CrossRef]

68. Guo, Y.; Qin, G.; Gu, H.; Qu, L.-J. Dof5. 6/HCA2, a Dof transcription factor gene, regulates interfascicular cambium formation and vascular tissue development in Arabidopsis. Plant Cell 2009, 21, 3518-3534. [CrossRef]

69. Kang, H.G.; Foley, R.C.; Oñate-Sánchez, L.; Lin, C.; Singh, K.B. Target genes for OBP3, a Dof transcription factor, include novel basic helix-loop-helix domain proteins inducible by salicylic acid. Plant J. 2003, 35, 362-372. [CrossRef]

70. Kang, H.G.; Singh, K.B. Characterization of salicylic acid-responsive, Arabidopsis Dof domain proteins: Overexpression of OBP3 leads to growth defects. Plant J. 2000, 21, 329-339. [CrossRef]

71. Romano, J.M.; Dubos, C.; Prouse, M.B.; Wilkins, O.; Hong, H.; Poole, M.; Kang, K.Y.; Li, E.; Douglas, C.J.; Western, T.L. AtMYB61, an R2R3-MYB transcription factor, functions as a pleiotropic regulator via a small gene network. New Phytol. 2012, 195, 774-786. [CrossRef] [PubMed]

72. Pastore, J.J.; Limpuangthip, A.; Yamaguchi, N.; Wu, M.-F.; Sang, Y.; Han, S.-K.; Malaspina, L.; Chavdaroff, N.; Yamaguchi, A.; Wagner, D. LATE MERISTEM IDENTITY2 acts together with LEAFY to activate APETALA1. Development 2011, 138, 3189-3198. [CrossRef] [PubMed] 
73. Gregis, V.; Sessa, A.; Dorca-Fornell, C.; Kater, M.M. The Arabidopsis floral meristem identity genes AP1, AGL24 and SVP directly repress class B and C floral homeotic genes. Plant J. 2009, 60, 626-637. [CrossRef] [PubMed]

74. Costanzo, E.; Trehin, C.; Vandenbussche, M. The role of WOX genes in flower development. Ann. Bot. 2014, 114, 1545-1553. [CrossRef] [PubMed] article distributed under the terms and conditions of the Creative Commons Attribution (CC BY) license (http://creativecommons.org/licenses/by/4.0/). 